\title{
Gender Prevalence of Urolithiasis in Albania: Our Data
}

\author{
Islam Mamica $^{1^{*}} \quad$ Gerta Halilaj $^{2} \quad$ Henri Kolani $^{3} \quad$ Rustem Celami $^{4} \quad$ Krenar Preza $^{5}$ \\ 1.Radiologist, Radiology Service, City Hospital, Mat, Albania \\ 2.Imagery Technician, Shefqet Ndroqi University Hospital and Lecturer at Aldent University, Tirana, Albania \\ 3.Surgeon, Surgery Service, Mother Teresa University Hospital Centre, Tirana, Albania \\ 4.Obstetrician and Gynecologist, American Hospital Albania; Professor, Aldent University, Tirana, Albania \\ 5.Radiologist, Professor, University of Medicine, Tirana, Albania
}

\begin{abstract}
Urolithiasis remain a problematic nosology in many countries and regions, however, there are studies that bring data on this nosology even in location, size and gender as well. We would like to bring in light our data in respect of gender prevalence of urolithiasis on our population study.

In our prospective study from 2011 to 2014 , of 200 subjects who were admitted to emergency service, respectively City Hospital of Mat, where 122 subjects were diagnosed with urolithiasis, and $67 \%$ of those subjects where males, or in other words 82 subjects, and $33 \%$ were female subjects, or in other words 40 subjects. In many studies there are data on gender and age as well in respect to urolithiasis. Our data, even in modest population would like to present and compare with literature and bring to the respective state authorities' attention for any future prospects.
\end{abstract}

Keywords: Urolithiasis, prevalence, gender, Albania

DOI: $10.7176 /$ ALST/87-01

Publication date: April $30^{\text {th }} 2021$

\section{Introduction}

According to previous studies previous decades on urolithiasis the gender prevalence of urolithiasis was more in man than women, however, recent studies show that this trend has changed due to many factors, so we are having today more women than man with urolithiasis. ${ }^{1,2}$

Some studies do loo ate dietary aspects, like amount of fluid intake, obesity, dietary intake in general. There are studies that show data on specific aspects like composition of the stone, age groups, regions, races, ethnicities and more other specific elements. ${ }^{3}$

Another problem we face in Albania, as in other nosologies and disciplines of medicines, we still do not have solid data on group age, regions of the country, gender, urolithiasis components, or solid diagnostic and treatment protocols.

A recent study was published on epidemiologic study of urolithiasis in seven countries of south east of Europe: S. E. G. U. R. 1 Study, like Greece, Turkey, Serbia, Northen Macedonia, Rumania, Bulgaria, Italy, unfortunately Albania was not part of this study project which would have bring solid data as e national reference on this respective nosology. ${ }^{4}$ The epidemiology of the urolithiasis nosology itself has changed constantly in respect to many other aspects like environment, geography regions, and ethnicity as well. So continuity of research on this matter is to bring up to date data and evidence based information where clinical practice is based on.

\section{Review of literature}

In recent Italian observational, descriptive, retrospective trial that was conducted in 2012, resulted thatprevalence of urolithiasis in Italy was $4.14 \%$ and was higher prevalend in males than females, respectively $4.53 \%$ versus $3.78 \%$ in females. ${ }^{5}$ There was seen a positive relation with increasing age. In respect to regions, the highest prevalence was seen in Campania around $6 \%$ and the highest incidence in region of Sicilia 3.15 per 1000 population.

It was shown in a study that the incidence of urolithiasis in Greece was estimated from $5-15 \%$, however there was stated that not data exist so it was only an estimation. ${ }^{6}$ Another study showed that prevalence of urolithisis in Greece was $15 \%{ }^{4}$

A national health survey in Serbia in 2013, in a cross sectional study that was carried out for national representative sample population of Serbia, $5.6 \%$ self reported kidney disease. ${ }^{7}$

According to study data, in Romani and Serbia was a higher rate of recurrent urilithiais than other 5 countries that were part of the study. ${ }^{4}$

\section{Results and discussion}

Our data obtained from e prospective study of e period of 3 years, 2011 - 2014, of a population study group 200 subjects where 122 were diagnosed with urolithiasis, 82 subjects of total population of 122 diagnosed subjects or 
of 200 total population that were seen in emergency department, concludes of $67 \%$ were male versus 40 subjects or $33 \%$ female, illustrated in table one and graph 1.

\begin{tabular}{|l|l|l|}
\hline Table 1. Urolithiasis distributions by gender & Percentage \% \\
\hline Gender & Nr. i cases & 67 \\
\hline Male & 82 & 33 \\
\hline Female & 40 & \\
\hline
\end{tabular}

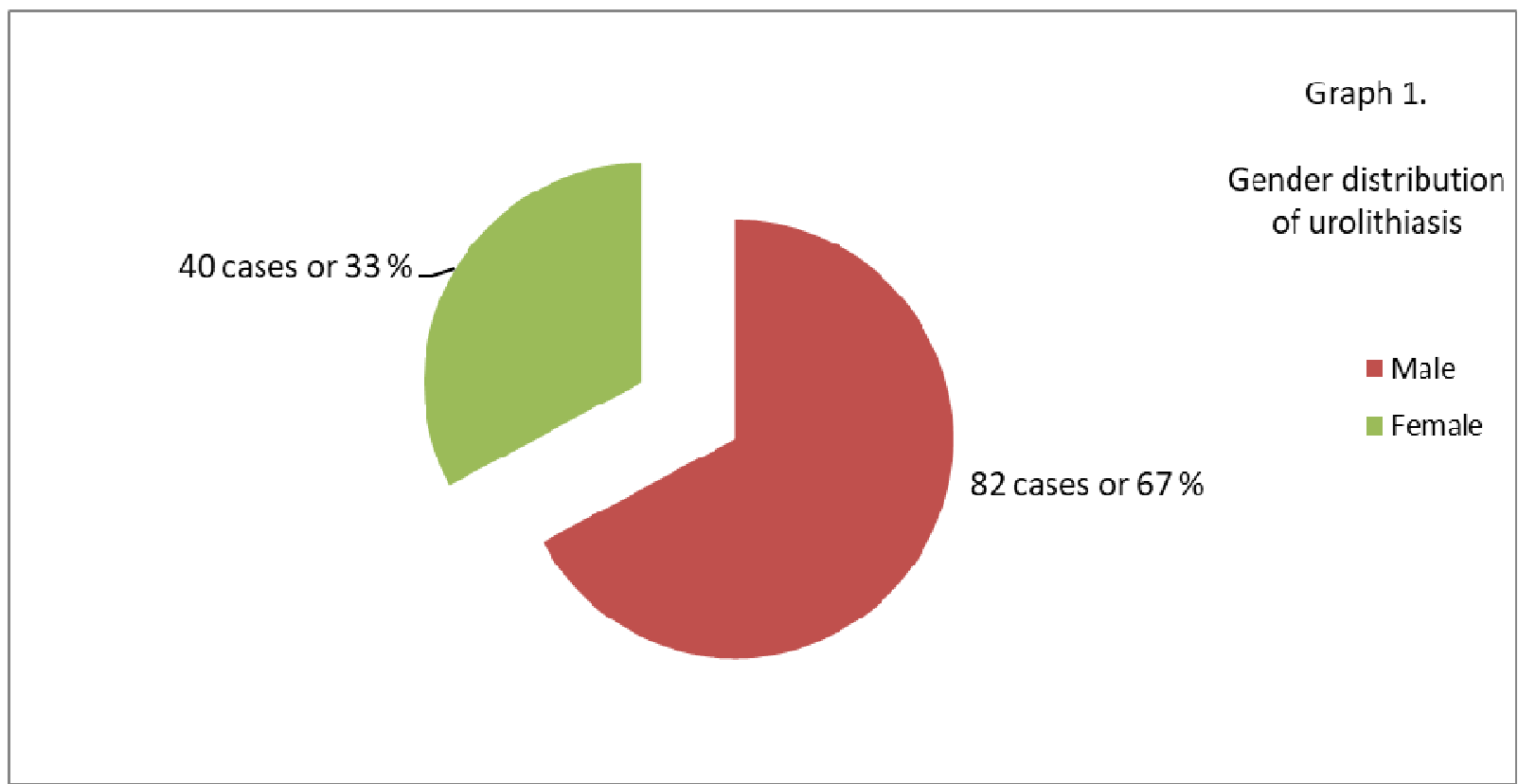

Our data correlates well with Italian and other regional studies where more male than female are affected by urolithiasis so far, however, recent studies show that a tendency towards a shift a gender related aspect of this nosology.

We need a national study to determine a solid evidence based professional opinion and work in guidelines in order to population awareness, prevention, early diagnosis and proper management of urolithiasis in our population.

\section{Conclusions}

Urolithiasis is the third most common urological problem, and it remains high prevalent, including Albania. Our data showed that males were affected almost two time more than females, $67 \%$ percent males versus $33 \%$ females. Gender affection of this nosology in most studies remain male more than female, however there are data that this is switching to be more prevalent in female than in male. In Albania we need to have more data in regards to any disease including urolithiasis based on our studies and our population based studies in order to project a healthier population and take all measures that are necessity.

\section{References}

1. Strope SA, Wolf JS Jr, Hollenbeck BK. Changes in gender distribution of urinary stone disease. Urology. 2010 Mar;75(3):543-6, 546.e1. doi: 10.1016/j.urology.2009.08.007. Epub 2009 Oct 24. PMID: 19854493; PMCID: PMC3410535.

2. WANG, Shu et al. Upper urinary tract stone compositions: the role of age and gender. Int. braz $j$ urol. [online]. 2020, vol.46, n.1 [cited 2021-03-11], pp.70-80. Available from: $<\mathrm{http}: / /$ www.scielo.br/scielo.php?script $=$ sci_arttext\&pid=S1677$55382020000100070 \& \operatorname{lng}=$ en\&nrm=iso $>$. $\quad$ Epub Jan 13, 6119. http://dx.doi.org/10.1590/s1677-5538.ibju.2019.0278.

2019.

ISSN

$1677-$

3. Rodgers AL. Race, ethnicity and urolithiasis: a critical review. Urolithiasis. 2013 Apr;41(2):99-103. doi: 10.1007/s00240-012-0516-9. Epub 2013 Jan 6. PMID: 23503870.

4. Karagiannis, Andreas \& Skolarikos, Andreas \& Alexandrescu, Emanuel \& Basic, Dragoslav \& Geavlete, Petrisor \& Maletta, Alessandro \& Muslumanoglu, A.Yaser \& Papatsoris, Athanasios \& Petkova, Kremena \& Saidi, S. \& Skakic, Aleksandar \& Saltirov, Iliya \& Sarica, Kemal \& Stavridis, Sotir \& Yilmaz, Omer \& Trinchieri, Alberto. (2017). Epidemiologic study of urolithiasis in seven countries of South-Eastern Europe: S.E.G.U.R. 1 study. Archivio Italiano di Urologia e Andrologia. 89. 173. 10.4081/aiua.2017.3.173. 
5. Prezioso, Domenico \& Illiano, Ester \& Piccinocchi, Gaetano \& Cricelli, Claudio \& Piccinocchi, Roberto \& Saita, Alberto \& Micheli, Carla \& Trinchieri, Alberto. (2014). Urolithiasis in Italy: An epidemiological study. Archivio Italiano di Urologia e Andrologia. 86. 99-102. 10.4081/aiua.2014.2.99.

6. Stamatiou KN, Karanasiou VI, Lacroix RE, Kavouras NG, Papadimitriou VT, Chlopsios C, Lebren FA, Sofras F. Prevalence of urolithiasis in rural Thebes, Greece. Rural Remote Health. 2006 Oct-Dec;6(4):610. Epub 2006 Dec 8. PMID: 17155848.

7. Jovic D, Dimkovic N, Rakocevic I, Boricic K, Atanasijevic D, Vasic M. Prevalence and factors associated with self-reported kidney disease among Serbian adults: Results of 2013 National Health Survey. PLoS One. 2018;13(9):e0203620. Published 2018 Sep 12. doi:10.1371/journal.pone.0203620. 